14.04

\title{
Поляронный перенос заряда в однородной Poly G/Poly C-цепочке в модели Пейрарда-Бишопа-Холстейна в постоянном электрическом поле
}

\author{
(C) А.Н. Коршунова, В.Д. Лахно \\ Институт математических проблем биологии РАН - филиал Федерального исследовательского центра „Институт \\ прикладной математики им. М.В. Келдыша РАН“, \\ Пущино, Московская область, Россия \\ e-mail: lak@impb.ru, alya@impb.ru
}

Поступило в Редакцию 11 декабря 2019 г.

В окончательной редакции 11 декабря 2019 г.

Принято к публикации 17 февраля 2020 г.

На основе модели Пейрарда-Бишопа-Холстейна проведены численные эксперименты, демонстрирующие возможность переноса заряда поляронами в однородной Poly G/Poly C-цепочке ДНК в постоянном электрическом поле. Показано, что в данной модели полярон может двигаться по цепочке с постоянной скоростью на большие расстояния для небольших значений напряженности электрического поля. С увеличением значения напряженности электрического поля равномерного движения заряда не наблюдается, заряд переходит в колебательный режим движения с блоховскими осцилляциями.

Ключевые слова: нанобиоэлектроника, нанопровода, молекулярные цепочки, поляроны, блоховские осцилляции.

DOI: 10.21883/JTF.2020.09.49686.397-19

\section{Введение}

Исследованию транспорта заряда в ДНК посвящено большое количество теоретических и экспериментальных работ [1-14]. Это связано, в первую очередь, с возможностью использования одномерных молекулярных цепочек в качестве нанопроводов в нанобиоэлектронных устройствах [15-19]. Считается, что основным носителем тока в однородных синтетических полинуклеотидных последовательностях является полярон [20-23].

Ранее в работе [24] нами было проведено численное моделирование движения заряда в холстейновской молекулярной цепочке в постоянном электрическом поле. Было показано существование равномерного движения заряда в цепочках с определенными значениями параметров для заданных значений напряженности электрического поля. В работе [24] было также показано, что, несмотря на простоту выбранной модели Холстейна $[25,26]$, в рассматриваемой системе могут реализоваться сложные динамические режимы, зависящие от всех выбранных параметров системы: от параметров цепочки, от величины напряженности электрического поля, от начального распределения заряда в цепочке.

В ранее используемой нами модели Холстейна, даже для однородной цепочки, при параметрах, соответствующих параметрам цепочки ДНК, полярон не двигался в электрическом поле. Однако в работе [27] нами показано, что в молекулярной цепочке, помещенной в сильное продольное электрическое поле, возможно образование новых типов возбуждений, которые способны переноситься по цепочке на большое расстояние (порядка тысяч нуклеотидных пар), приближенно сохраняя свою форму. Эти возбуждения, или неравновесные поляроны, формируются из специально заданных начальных условий. Также, в проведенных ранее исследованиях блоховские осцилляции полярона и особенности стационарного движения полярона по цепочке в постоянном электрическом поле были рассмотрены в работах $[28,29]$.

В настоящей работе мы используем более детальную модель ДНК, модель Пейрарда-Бишопа-Холстейна (ПБХ) [30-33]. В настоящее время считается, что по сравнению с простой моделью Холстейна модель ПБХ лучше описывает динамические свойства ДНК.

Ранее в работах [34-36] нами были изучены особенности движения заряда в однородных молекулярных полинуклеотидных цепочках конечной длины при отсутствии внешнего электрического поля. Исследования проводились в цепочках на основе моделей Пейрарда-Бишопа-Холстейна и Пейрарда-Бишопа-Доксуа-Холстейна. В работах $[34,35]$ для моделирования переноса заряда по цепочке, в начальный момент времени в цепочку вносился полярон. В статье [34] было показано, что полярон, помещенный в начальный момент времени не в центр цепочки, приобретает способность двигаться. В работе [35] представлены численные эксперименты, демонстрирующие возможность переноса заряда поляронами в однородной незамкнутой $G / C$-цепочке ДНК за счет взаимодействия с локализованными возбуждениями при отсутствии электрического поля в цепочке. В работе [36] в начальный момент времени в цепочку вносился не полярон, а заряд, локализованный в области определенного числа соседних сайтов, смещенных из положений равновесия. Было показано, что для лучшего запуска движения заряда, задаваемые начальные условия 
должны соответствовать параметрам полярона, который образуется в цепочке при выбранных параметрах.

\section{1. Математическая модель}

Исследование поляронного переноса заряда в однородной Poly $G /$ Poly $C$-цепочке будем проводить в рамках квантово-классической модели, которая является комбинацией модели Холстейна $[25,26]$ и модели Пейрарда-Бишопа [30]. В этом случае простое описание динамики возмущений в цепочке сайтов, используемое в модели Холстейна, заменено на предложенное в работе [30], где для описания взаимодействия нуклеотидов в паре используется нелинейный потенциал Морзе. Подробно это описано в работах [37,38].

Гамильтониан модели записывается в виде:

$$
\begin{aligned}
\hat{H} & =-\sum_{n} v(|n\rangle\langle n-1|+| n\rangle\langle n+1|)+\chi \sqrt{2} \sum_{n} y_{n}|n\rangle\langle n| \\
& +\sum_{n}\left[\frac{1}{2} m \dot{y}_{n}^{2}+\frac{k}{2}\left(y_{n}-y_{n-1}\right)^{2}+D\left(e^{-\sigma y_{n}}-1\right)^{2}\right], \quad
\end{aligned}
$$

где $v$ - матричный элемент перехода заряда между соседними сайтами (нуклеотидными парами), $\chi-$ константа связи внешнего заряда и цепочки заряда, $m$ масса нуклеотида, $k$ - параметр жесткости стэкингового потенциала. Переменные $y_{n}$ описывают отклонения нуклеотидов $n$-ой пары от их равновесных положений. Связь нуклеотидов в каждой паре описывается нелинейным потенциалом Морзе:

$$
D\left(e^{-\sigma y_{n}}-1\right)^{2},
$$

где положительные параметры потенциала Морзе $D$ и $\sigma$ задают глубину его потенциальной ямы и коэффициент жесткости.

Перейдем к безразмерным переменным с помощью соотношений: $q_{n}=\sigma y_{n}, \tilde{t}=\omega_{M} t, \omega_{M}=\left(2 \sigma^{2} D / m\right)^{1 / 2}-$ частота линейных колебаний Морзе осциллятора, $\omega_{b o n d}^{2}=k /\left(2 \sigma^{2} D\right)-$ безразмерная частота линейных колебаний нуклеотида, $\chi_{e l}=\sqrt{2} \chi / \hbar \omega_{M} \sigma, \chi_{h}=\sqrt{2} \chi / 2 \sigma D$, $\tau_{e}=v / \hbar \omega_{M}$.

Динамика квантовой частицы в классической цепочке описывается системой нелинейных дифференциальных уравнений, которая в безразмерных переменных имеет вид

$$
\begin{gathered}
\dot{c}_{n}=-i \tau_{e}\left(c_{n-1}+c_{n+1}\right)-i \chi_{e l} q_{n} c_{n}-i \lambda \widetilde{E} n c_{n}, \\
\ddot{q}_{n}+\Gamma \dot{q}_{n}=e^{-q_{n}}\left(e^{-q_{n}}-1\right)+\omega_{b o n d}^{2} \\
\times\left(q_{n-1}-2 q_{n}+q_{n+1}\right)-\chi_{h}\left|c_{n}\right|^{2},
\end{gathered}
$$

где $c_{n}$ - амплитуды вероятности локализации заряда на $n$-ом сайте, $\tau_{e}$ - отношение характерных времен эволюции волновой функции электрона и динамики возмущений в решетке, $\chi_{e l}$ и $\chi_{h}-$ безразмерные параметры связи электрона и решетки, $q_{n}$ - нормированное смещение, $\omega_{\text {bond }}-$ нормированная частота колебаний сайтов в решетке, $\Gamma-$ коэффициент трения, $\lambda=e E_{0} a / \hbar \omega_{M}$, $E=\widetilde{E} E_{0}, E_{0}=10^{4} \mathrm{~V} / \mathrm{cm}, E-$ напряженность электрического поля, $a-$ постоянная решетки, $\tilde{t}=\omega_{M} t-$ безразмерное время. (3) - дискретные уравнения Шредингера для электрона, (4) - уравнения движения нуклеотидных пар. Цепочка рассматривается однородная, незамкнутая, т. е. цепочка имеет два конца.

Система нелинейных дифференциальных уравнений (3), (4) решается методами (a) - типа Рунге-Кутта 4-го порядка и (b) - явного 4-шагового метода Адамca-Бэшфорта на этапе предсказания и расчетом поправки 3-шаговым методом Адамса-Мултона. Расчеты выполнялись с использованием вычислительных средств МСЦ РАН.

\section{2. Выбор параметров цепочки для моделирования движения полярона в электрическом поле}

Для моделирования движения полярона в постоянном электрическом поле мы помещаем в цепочку (в начальный момент времени) полярон, соответствующий стационарному решению уравнений $(3),(4)$ в отсутствие внешнего поля. Центр полярона помещается на сайт цепочки с номером $n_{0}$. Начальные значения выбирались в виде обратного гиперболического косинуса:

$$
\begin{gathered}
\left|c_{n}(0)\right|=\frac{\sqrt{2}}{4} \sqrt{\frac{\chi_{e l} \cdot \chi_{h}}{\tau_{e}}} \operatorname{ch}^{-1}\left(\frac{\left(\chi_{e l} \cdot \chi_{h}\right)\left(n-n_{0}\right)}{4 \tau_{e}}\right), \\
q_{n}(0)=-\left|c_{n}(0)\right|^{2} \cdot \chi_{h}, \quad \dot{q}_{n}(0)=0 .
\end{gathered}
$$

Длина цепочки выбирается такой, чтобы полярон не оказался слишком близко к концу цепочки до окончания вычислений. Значение $n_{0}$ (центр начального обратного гиперболического косинуса) в (5) выбирается так, чтобы на начальном этапе вычислений полярон находился достаточно далеко от концов цепочки. Поле включается „мгновенно“ в начальный момент времени.

Численные значения параметров, используемые в моделировании, выбраны в соответствии с параметрами ПБХ модели в работах $[37,38,30]$. Значения параметров выбирались с учетом результатов, полученных в экспериментах. Таким образом, были выбраны следующие значения параметров: $D=0.04 \mathrm{eV}, \sigma=4.45 \AA^{-1}, k=$ $=0.06 \mathrm{eV} / \AA^{2}, m=300$ a.e.m., $\chi=0.13 \mathrm{eV} / \AA, v=0.084 \mathrm{eV}$, тогда $\omega_{M}=7.1 \cdot 10^{-12} \mathrm{~s}^{-1}$.

Следовательно, будем считать, что цепочке ДНК соответствуют следующие безразмерные значения параметров: $\tau_{e}=18, \chi_{e l}=8.5, \chi_{h}=0.5, \omega_{\text {bond }}=0.2, \Gamma=0.084$, $\lambda=0.074$. При таких параметрах цепочки в ПБХ модели полярон располагается на достаточно большом количестве сайтов (рис. 1 для $\tau_{e}=18$ ), его характерный размер:

$$
d(t)=\sum\left|c_{n}(\tilde{t})\right|^{2} / \sum\left|c_{n}(\tilde{t})\right|^{4}=1 / \sum\left|c_{n}(\tilde{t})\right|^{4},
$$




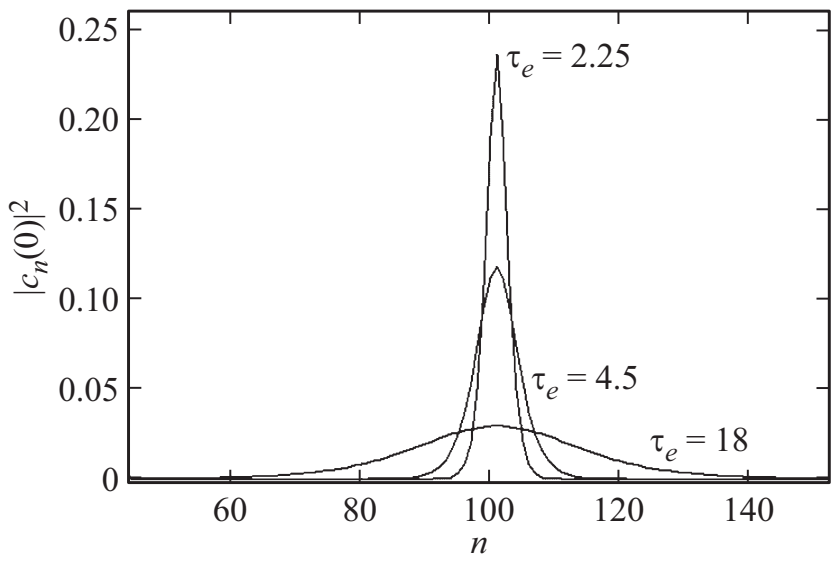

Рис. 1. Графики функции $\left|c_{n}(0)\right|^{2}$ вида (5) в отсутствие электрического поля для различных значений параметра $\tau_{e}$.

в отсутствие электрического поля $d(t) \approx 52$, в отличие от полярона в модели Холстейна. В модели Холстейна полярон в цепочке с параметрами, соответствующими цепочке ДНК, располагался всего на нескольких сайтах $(\approx 2)$ и без специальных условий для запуска движения в электрическом поле не двигался.

На рис. 1 представлены графики функции $\left|c_{n}(0)\right|^{2}$ вида (5) в отсутствие электрического поля для $\tau_{e}=18$, 4.5 и 2.25. Далее в работе будут представлены примеры моделирования движения полярона в цепочках с указанными значениями параметра $\tau_{e}$, значения других параметров цепочки изменяться не будут, будут использоваться их значения, указанные выше.

Тот факт, что для ПБХ модели с указанными значениями параметров для $\tau_{e}=18$ полярон имеет большой характерный размер, приводит к предположению, что такое состояние может двигаться во внешнем электрическом поле. То есть мы можем поместить такой стационарный полярон в цепочку, не меняя его формы, не создавая никаких дополнительных условий для запуска движения, подключить электрическое поле и увидеть какое-то премещение полярона по цепочке. Вычисления показывают, что такой широкий полярон может двигаться равномерно только при очень маленьких значениях напряженности электрического поля $\widetilde{E}$. Например, при значении $\widetilde{E}=0.01$ полярон начинает совершать блоховские осцилляции.

\section{3. Движение полярона в электрическом поле}

На рис. 2 представлены графики функции $X \widetilde{(t)}$ для различных значений напряженности электрического поля $\widetilde{E}$

$$
X(\widetilde{t})=\sum_{n}\left|c_{n}(\widetilde{t})\right|^{2} \cdot n,
$$

где функция $X(\widetilde{t})$ описывает движение центра масс заряда. В представленных далее примерах сайты в цепочках были пронумерованы слева направо. Так как мы выбирали значения $\widetilde{E}>0$, значения $n_{0}$ в (5) были выбраны вблизи правого конца цепочки. Таким образом, поляроны или возбуждения двигаются по цепочке в направлении поля справа налево.

В примере, показанном на рис. 2, полярон вида (5) в начальный момент времени находится на сайте c номером $n_{0}=22000$, длина цепочки $N=24000$ сайтов. Для всех заданных значений напряженности электрического поля $\widetilde{E}$ наблюдается колебательный режим движения заряда. Графики функции $X(\widetilde{t})$, представленные на рис. 2, показывают хорошее соответствие численных и теоретических значений периода блоховских осцилляций и максимальной амплитуды блоховских осцилляций. Период блоховских осцилляций $\widetilde{T}_{B L} \approx 13.3 \cdot 2 \pi / \widetilde{E} \approx 83.56 / \widetilde{E}$, максимальная амплитуда блоховских осцилляций $A_{B L} \approx 4 \tau_{e} / \lambda \widetilde{E}$. Для заданных значений напряженности электрического поля $\widetilde{E}=0.05$, $0.06,0.07,0.08,0.09$, теоретические значения периода блоховских осцилляций $\widetilde{T}_{B L} \approx 83.56 / \widetilde{E}$ примерно равны 1671, 1393, 1194, 1045, 928 соответственно. Максимальная амплитуда блоховских осцилляций $A_{B L} \approx 4 \tau_{e} / \lambda \widetilde{E}$ для $\widetilde{E}=0.05$ примерно равна 19460 , для $\widetilde{E}=0.08-12162$, для $\widetilde{E}=0.09-10811$.

Таким образом, на рис. 2 мы наблюдаем блоховские осцилляции заряда для всех заданных значений напряженности электрического поля.

Ранее в работах $[24,29]$ для холстейновского полярона было показано, что движение заряда с постоянной скоростью возможно для небольших значений напряженности электрического поля. С увеличением значения напряженности электрического поля заряд переходит в колебательный режим движения с блоховскими осцилляциями.

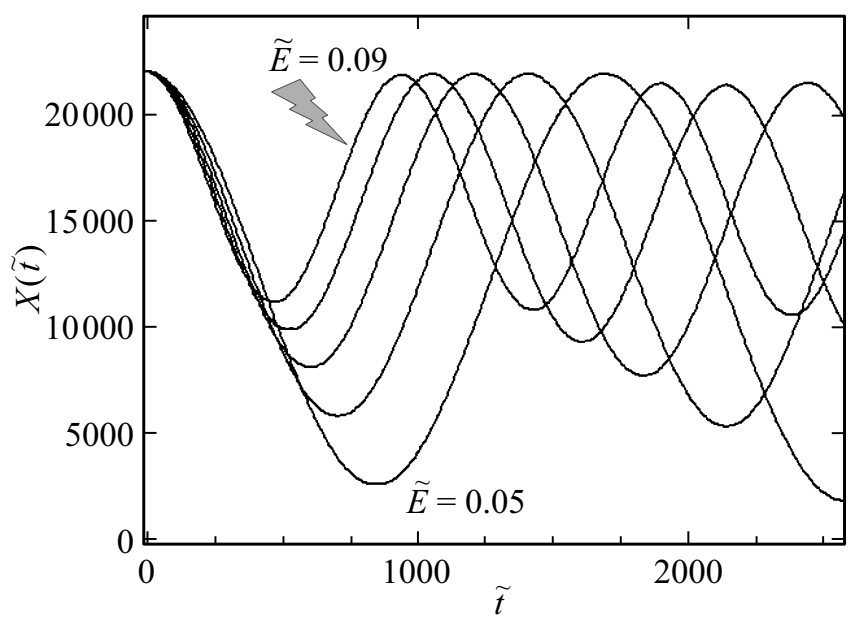

Рис. 2. Графики функции $X(\widetilde{t})$ для различных значений напряженности электрического поля $\widetilde{E}=0.05,0.06,0.07,0.08$, 0.09 . Длина цепочки $N=24000$ сайтов. Верхний график соответствует значению $\widetilde{E}=0.09$, нижний график соответствует значению $\widetilde{E}=0.05$. Значение параметра $\tau_{e}=18$. 


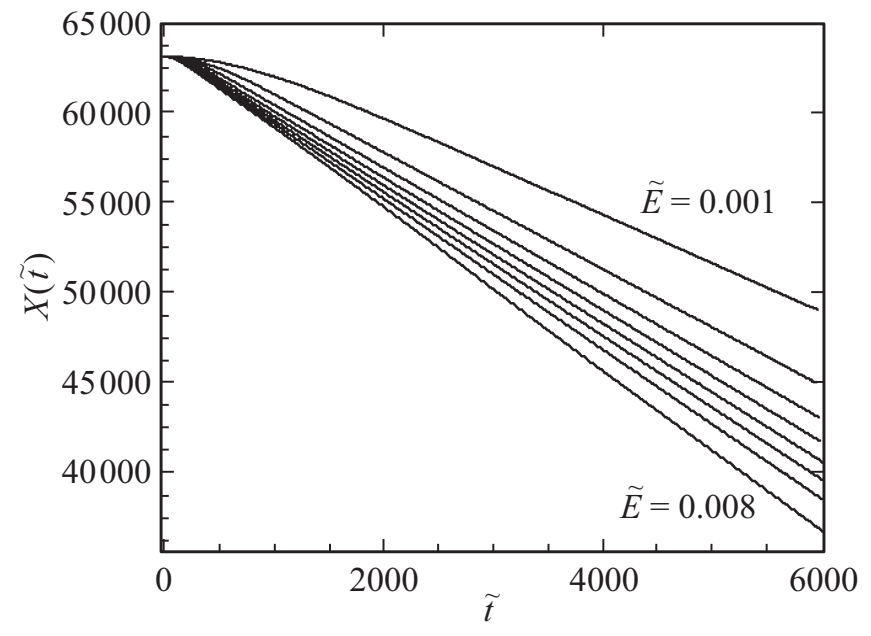

Рис. 3. График функции $X(\widetilde{t})$ для различных значений напряженности электрического поля $\widetilde{E}=0.001,0.002,0.003$, $0.004,0.005,0.006,0.007,0.008$. Длина цепочки $N=65000$ сайтов. Верхний график соответствует значению $\widetilde{E}=0.001$, нижний график соответствует значению $\widetilde{E}=0.008$. Значение параметра $\tau_{e}=18$.

В примере, показанном на рис. 3, моделирование движения заряда по цепочке было проведено для меньших значений напряженности электрического поля. В начальный момент времени полярон вида (5) находится на сайте с номером $n_{0}=63000$, длина цепочки $N=65000$ сайтов. Графики функции $X(\widetilde{t})$, представленные на рис. 3 выглядят прямыми линиями, это может указывать на равномерное движение заряда по цепочке. Но в данном случае утверждать, что мы видим графики, показывающие равномерное движение, нельзя.

Безразмерное время вычислений, показанное на рис. $3, \widetilde{t} \approx 6000$. Сравним эту величину с периодом блоховских осцилляций для выбранных в примере на рис. 3 значений напряженности электрического поля. В примере на рис. 3 теоретические значения периода блоховских осцилляций $\widetilde{T}_{B L} \approx 83.56 / \widetilde{E}$ для заданных значений напряженности электрического поля $\widetilde{E}=0.001$, $0.002,0.003,0.004,0.005,0.006,0.007,0.008$, примерно равны $83560,41780,27853,20890,16712,13927$, 11937,10445 соответственно. То есть для всех представленных графиков время вычислений $(t \approx 6000)$ меньше продолжительности даже одного блоховского периода.

Таким образом, если равномерное движение существует, то оно может наблюдаться при значениях $\widetilde{E}$ порядка $\widetilde{E}=0.001$ и меньше. Для $\widetilde{E}=0.001$ один безразмерный период блоховских осцилляций $\widetilde{T}_{B L} \approx 83560$, а максимальная блоховская амплитуда $A_{B L} \approx 973000$. Таким образом, для того, чтобы выполнить моделирование движения полярона в цепочке с параметрами ДНК всего лишь в течение одного блоховского периода, нужно взять цепочку длиной около $1.5 \cdot 10^{6}$ сайтов и провести вычисление на протяжении $\tilde{t} \approx 10^{5}$. Из этого следует, что прежде, чем моделировать равномерное движение полярона в цепочке с параметрами ДНК, целесообразно провести вычислительные эксперименты для цепочек с такими параметрами, которые требуют меньших затрат. И затем на примере модельных цепочек проводить вычисления для цепочек с параметрами ДНК.

Поскольку нас прежде всего интересует равномерное движение полярона в цепочке с параметрами ДНК, мы возьмем для модельных цепочек те же параметры, что и для цепочки ДНК, кроме параметров $\tau_{e}$ (отношение характерных времен эволюции волновой функции электрона и динамики возмущений в решетке).

Для того чтобы утверждать, что какой либо график функции $X(t)$ указывает на равномерное движение полярона, нужно провести вычисления на протяжении нескольких блоховских периодов. Хотя при определенных условиях нескольких периодов может быть недостаточно, ниже мы это покажем. Поэтому, несмотря на то, что мы моделируем не блоховские осцилляции а равномерное движение полярона, нам необходимо учитывать основные характеристики блоховских осцилляций, а именно: значение периода блоховских осцилляций $\widetilde{T}_{B L} \approx 13.3 \cdot 2 \pi / \widetilde{E} \approx 83.56 / \widetilde{E}$, максимальную амплитуду блоховских осцилляций $A_{B L} \approx 4 \tau_{e} / \lambda \widetilde{E}$ и максимальную скорость блоховских осцилляций $\widetilde{V}_{B L}^{\max } \approx 2 \tau_{e}$. Поскольку величина максимальной блоховской амплитуды прямо пропорциональна величине $\tau_{e}$, мы возьмем для моделирования значения $\tau_{e}$ в четыре и в восемь раз меньше значения $\tau_{e}$ соответствующего цепочке ДНК, а именно $\tau_{e}=4.5$ и 2.25. На рис. 1 представлены графики функции $\left|c_{n}(0)\right|^{2}$ вида (5) в отсутствие электрического поля для $\tau_{e}=4.5$ и 2.25. Для выбранных значений $\tau_{e}$ соответствующие поляроны уже не такие широкие, как для $\tau_{e}=18$, следовательно, они могут двигаться равномерно при бо́льших значениях напряженности электрического поля $\widetilde{E}$. Таким образом, объем вычислений сокращается еще и за счет увеличения требуемых значений $\widetilde{E}$, поскольку период и максимальная амплитуда блоховских осцилляций обратно пропорциональны значению $\widetilde{E}$.

Рис. 4 иллюстрирует следующий вычислительный эксперимент. В начальный момент времени в цепочку длиной 18000 сайтов помещается полярон вида (5) для $\tau_{e}=4.5$ с центром на сайте с номером 16000 . Поле включается мгновенно в начальный момент времени. Моделирование движения полярона в поле проводилось для восьми различных значений напряженности электрического поля $\widetilde{E}=0.05,0.06,0.07,0.08,0.09,0.1$, $0.11,0.12$. Безразмерное время вычислений графиков, представленных на рис. 4 , примерно равно $\widetilde{t} \approx 11000$.

Как и в предыдущем примере, сравним это время с периодом блоховских осцилляций для выбранных значений напряженности электрического поля. Теоретические значения периода блоховских осцилляций $\widetilde{T}_{B L} \approx 83.56 / \widetilde{E}$ для заданных значений напряженности электрического поля $\widetilde{E}=0.05,0.06,0.07,0.08,0.09,0.1,0.11,0.12$ примерно равны 1671, 1393, 1194, 1045, 928, 836, 760, 696 соответственно. Таким образом, для всех представленных графиков, время вычислений $(\widetilde{t} \approx 11000)$ 


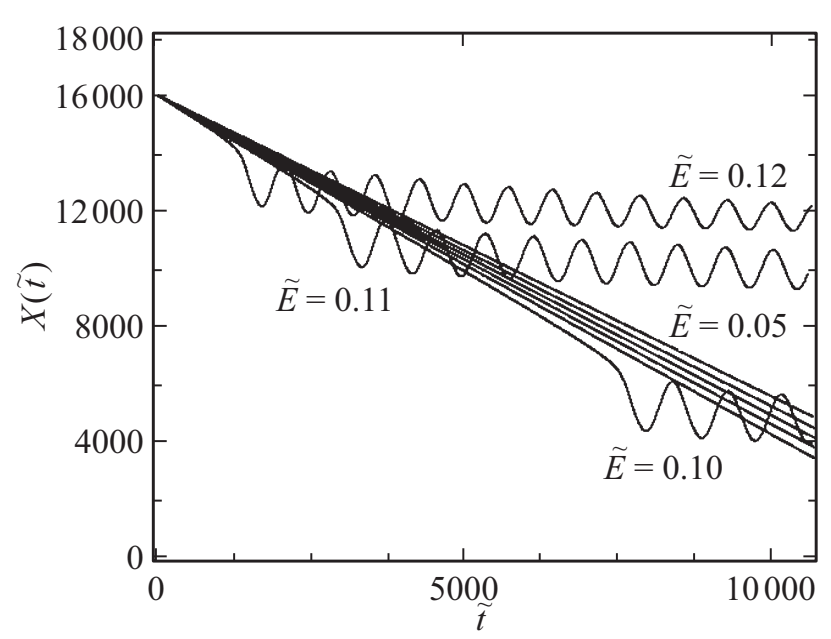

Рис. 4. Графики функции $X(\widetilde{t})$ для различных значений напряженности электрического поля $\widetilde{E}=0.05,0.06,0.07,0.08,0.09$, $0.1,0.11,0.12$. Длина цепочки $N=18000$ сайтов. Значение параметра $\tau_{e}=4.5$.

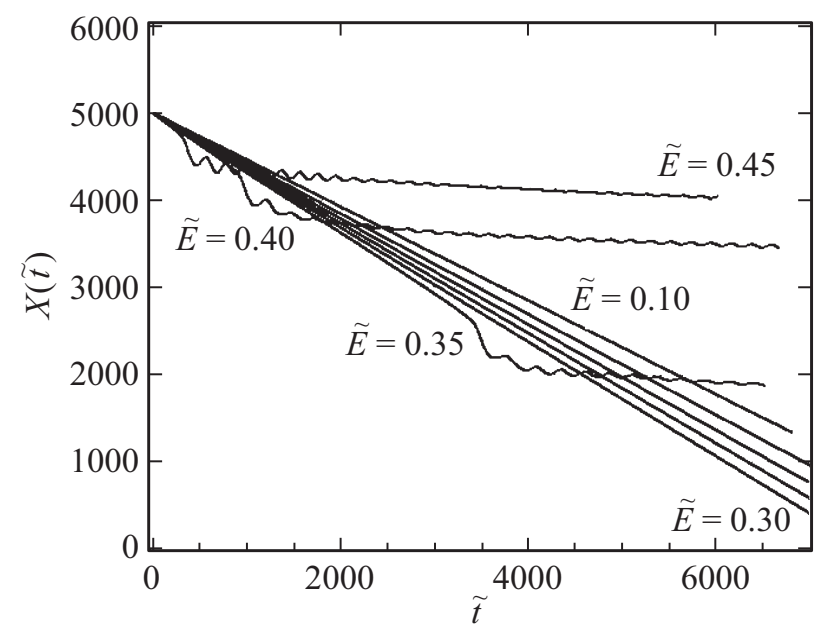

Рис. 5. Графики функции $X(\widetilde{t})$ для различных значений напряженности электрического поля $\widetilde{E}=0.1,0.15,0.2,0.25$, $0.3,0.35,0.4,0.45$. Длина цепочки $N=6000$ сайтов. Значение параметра $\tau_{e}=2.25$.

значительно превышает продолжительность блоховского периода для любого указанного значения напряженности электрического поля $\widetilde{E}$. Время вычисления графика для $\widetilde{E}=0.05$ больше продолжительности шести блоховских периодов, для $\widetilde{E}=0.12-$ больше пятнадцати блоховских периодов.

График функции $X(\widetilde{t})$ для $\widetilde{E}=0.12$ на рис. 4 показывает, что после начала движения полярон движется по цепочке практически равномерно на протяжении примерно двух блоховских периодов. Затем полярон резко теряет свою начальную форму и переходит в колебательный режим движения с блоховскими осцилляциями. При этом численный период блоховских осцилляций очень близок к теоретическому. Рис. 4 наглядно показывает, что с уменьшением значения напряженности электри- ческого поля $\widetilde{E}$, период почти равномерного движения полярона до момента его перехода в колебательный режим движения значительно возрастает. Для $\widetilde{E}=0.1$ полярон двигается почти равномерно в течение примерно девяти блоховских периодов, и только затем переходит в колебательный режим движения с блоховскими осцилляциями, соответствующими заданному значению напряженности электрического поля.

Для значений напряженности электрического поля $\widetilde{E}<0.1$, графики функции $X(t)$ на рис. 4 выглядят прямыми линиями в течение показанного времени вычислений. Но, учитывая поведение полярона в данном примере для напряженности электрического поля $\widetilde{E}=0.1,0.11,0.12$, можно предположить, что и для меньших значений $\widetilde{E}$, в какой-то момент времени полярон потеряет свою первоначальную форму и перейдет в колебательный режим движения. Но из графиков $X(t)$ на рис. 4 также следует, что период почти равномерного движения полярона после начала движения значительно и нелинейно возрастает с уменьшением значения $\widetilde{E}$. Поэтому можно утверждать, что для небольших значений напряженности электрического поля полярон может двигаться по цепочке равномерно на очень большие расстояния.

На рис. 5 представлены результаты аналогичных вычислений для цепочки с параметром $\tau_{e}=2.25$. Полярон в этой цепочке значительно у́же поляронов в цепочках с параметрами $\tau_{e}=4.5$ и 18 (рис. 1). Поскольку полярон вида (5) для $\tau_{e}=2.25$ значительно у́же ранее рассмотренных, то для моделирования движения полярона мы можем задать бо́льшие значения напряженности электрического поля: $\widetilde{E}=0.1,0.15,0.2,0.25,0.3,0.35$, $0.4,0.45$. В данном случае выбрана цепочка длиной 6000 сайтов, начальный полярон вида 5 был помещен на сайт с номером 5000. Поле включается мгновенно в начальный момент времени.

Как и в примере на рис. 4,5 для пяти ме́ньших значений напряженности поля $\widetilde{E}=0.1,0.15,0.2,0.25,0.3$ графики функций $X(\widetilde{t})$ выглядят прямыми линиями. Для бо́льших значений напряженности электрического поля поведение полярона такое же, как и в примере на рис. 4. Продолжительность времени вычислений на рис. 5 примерно равна $\tilde{t} \approx 7000$. Период блоховских осцилляций для $\widetilde{E}=0.45$ примерно равен $\widetilde{T}_{B L} \approx 186$, для $\widetilde{E}=0.3$ $-\widetilde{T}_{B L} \approx 278$. Следовательно, график функции $X(\widetilde{t})$ для $\widetilde{E}=0.45$ на рис. 5 показан (для $\widetilde{t} \approx 6000$ ) на протяжении более 32 блоховских периодов, для $\widetilde{E}=0.3-$ на протяжении более 25 блоховских периодов (для $\widetilde{t} \approx 7000$ ).

Покажем, как выглядят функции амплитуд вероятностй локализации заряда на $n$-ом сайте $\left|c_{n}(\tilde{t})\right|^{2}$ в процессе движения заряда в электрическом поле в небольшой начальный период времени.

График функции $X(\widetilde{t})$ для значения напряженности электрического поля $\widetilde{E}=0.45$ показан на рис. 5 для большого периода времени. На рис. 6 показаны графики функций $X(\tilde{t})$ и $\left|c_{n}(\tilde{t})\right|^{2}$ при движении заряда в электрическом поле напряженностью $\widetilde{E}=0.45$. Центр 


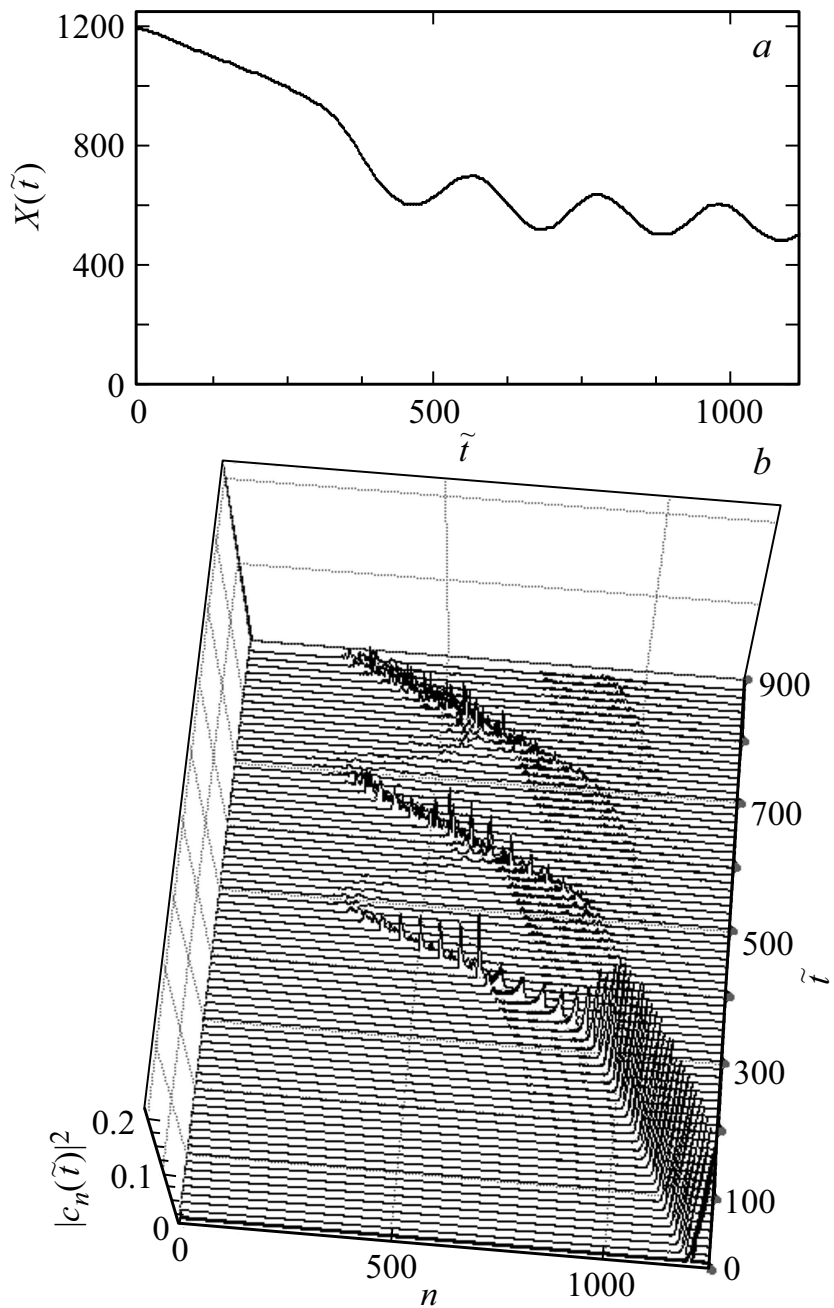

Рис. 6. Графики функций $X(\widetilde{t})$ и $\left|c_{n}(\tilde{t})\right|^{2}$ при движении заряда в электрическом поле напряженностью $\widetilde{E}=0.45$. Длина цепочки $N=1250$ сайтов. Значение $\tau_{e}=2.25$.

заряда в начальный момент времени находится на сайте с номером $n_{0}=1200$ в цепочке длиной 1250 сайтов. Графики функций $X(\widetilde{t})$ и $\left|c_{n}(\tilde{t})\right|^{2}$ на рис. 6 наглядно показывают, что для заданного значения напряженности электрического поля начальный полярон медленно „оседает“, двигаясь по цепочке почти равномерно, затем резко теряет свою первоначальную форму и переходит в колебательный режим движения с блоховскими осцилляциями.

На рис. 7 показаны графики функций $X(\widetilde{t})$ и $\left|c_{n}(\tilde{t})\right|^{2}$ при достаточно большом значении напряженности электрического поля $\widetilde{E}=0.7$. В начальный момент времени заряд находится на сайте с номером $n_{0}=650$ в цепочке длиной 700 сайтов. В этом примере заряд сразу после включения поля теряет свою первоначальную форму и переходит в колебательный режим движения. Теоретический период блоховских осцилляций для $\widetilde{E}=0.7$ примерно равен $\widetilde{T}_{B L} \approx 119$, это значение достаточно близко к численному периоду осцилляций, который демонстрируют графики функций $X(\widetilde{t})$ и $\left|c_{n}(\tilde{t})\right|^{2}$ на рис. 7.
Анализируя графики функции $X(\widetilde{t})$ на рис. 4 и 5, можно предположить, что полярон движется какое-то время почти с постоянной скоростью для небольших значений напряженности электрического поля $\widetilde{E}$, а затем деформируется и переходит в колебательный режим движения. Причем, чем меньше значение $\widetilde{E}$, тем дольше полярон движется с постоянной скоростью. И для очень маленьких значений поля продолжительность такого движения с постоянной скоростью может стремиться к бесконечно большим значениям.

В примерах, показанных на рис. 5 и 8 движение полярона моделируется в одинаковых цепочках, примеры отличаются только выбранными значениями напряженности электрического поля. Причем, в примере на рис. 8 моделирование движения полярона проводилось для тех же значений напряженности электрического поля, что и на рис. 4 при $\tau_{e}=4.5: \widetilde{E}=0.05,0.06,0.07,0.08,0.09,0.1$, $0.11,0.12$.

Рис. 8 показывает следующий вычислительный эксперимент. В цепочку длиной 13000 сайтов помещается

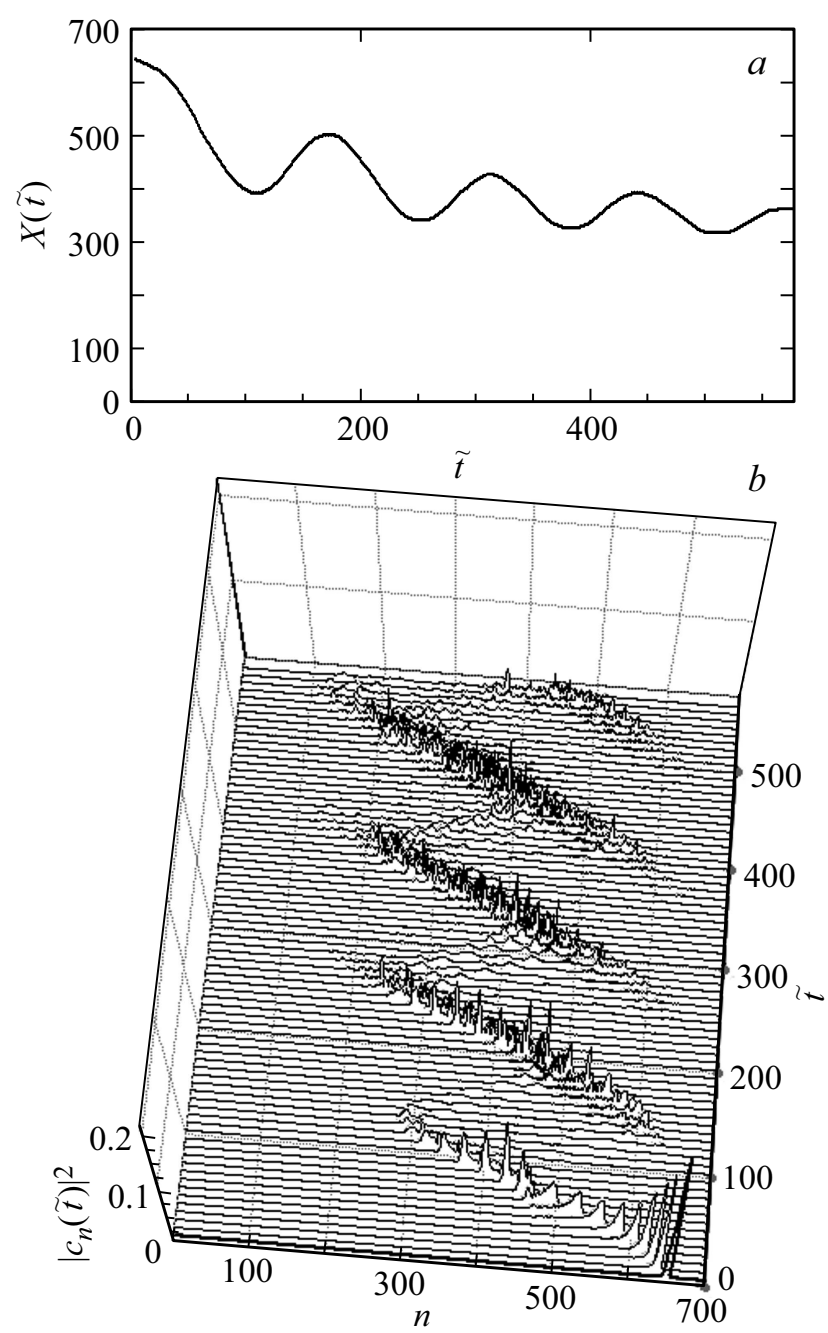

Рис. 7. Графики функций $X(\widetilde{t})$ и $\left|c_{n}(\tilde{t})\right|^{2}$ при движении заряда в электрическом поле напряженностью $\widetilde{E}=0.7$. Длина цепочки $N=700$ сайтов. Значение $\tau_{e}=2.25$. 


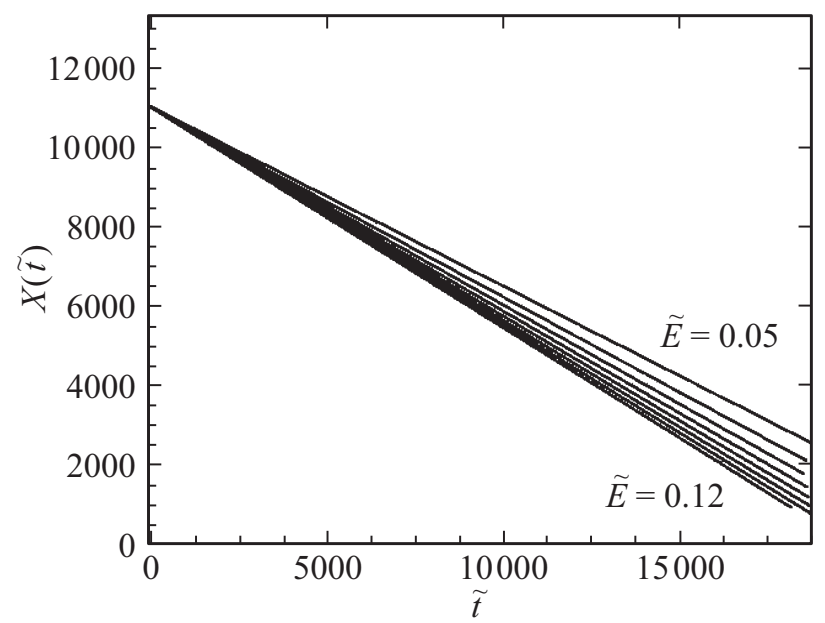

Рис. 8. Графики функции $X(t)$ для различных значений напряженности электрического поля $\widetilde{E}=0.05,0.06,0.07,0.08,0.09$, $0.1,0.11,0.12$. Длина цепочки $N=13000$ сайтов. Значение $\tau_{e}=2.25$.

полярон вида (5) для $\tau_{e}=2.25$ с центром на сайте с номером 11000 . Поле включается мгновенно в начальный момент времени. Продолжительность вычислений графиков на рис. 8 примерно равна $\widetilde{t} \approx 19000$.

Теоретические значения периода блоховских осцилляций $\widetilde{T}_{B L} \approx 83.56 / \widetilde{E}$ зависят от значения напряженности электрического поля $\widetilde{E}$. Так как в примере на рис. 8 мы выбрали те же значения $\widetilde{E}$, что в примере на рис. 4 , то и блоховские периоды в обоих случаях одинаковые. То есть в этом примере период блоховских осцилляций для $\widetilde{E}=0.05$ примерно равен $\widetilde{T}_{B L} \approx 1671$, для $\widetilde{E}=0.12-$ $\widetilde{T}_{B L} \approx 696$. Из чего следует, что на рис. 8 график функции $X(t)$ для $\widetilde{E}=0.05$ показан на протяжении более 11 блоховских периодов, для $\widetilde{E}=0.12-$ на протяжении более 27 блоховских периодов. Поскольку безразмерное время вычислений на рис. 8 больше, чем на рис. 4 , то каждый график функции $X(t)$ вычислен для большего количества блоховских периодов.

Из рис. 8 следует, что для всех значений $\widetilde{E}$ в течение показанного времени вычислений $(\widetilde{t} \approx 19000)$ полярон движется по цепочке с постоянной скоростью, поскольку для всех значений $\widetilde{E}$ графики функции $X(t)$ выглядят прямыми линиями.

\section{Заключение}

Ранее в работах $[24,27,29]$ нами было показано существование равномерного движения заряда в цепочках с определенными значениями параметров для заданных значений напряженности электрического поля на основе модели Холстейна. В работе для моделирования движения заряда в полинуклеотидной цепочке мы использовали более детальную модель ДНК, модель ПБХ [30-33]. В наших исследованиях мы использовали численные значения параметров для ПБХ модели со- ответствующие параметрам цепочки ДНК, указанные в работах $[37,38,30]$. В разд. 2 данной статьи мы указали безразмерные значения этих параметров.

Численное моделирование движения заряда в цепочке с такими параметрами показало, что равномерное движение заряда может существовать при значениях напряженности электрического поля $\widetilde{E}$ меньше безразмерного значения $\widetilde{E}<0.001$. Это безразмерное значени соотвтствует размерному значению напряженности электрического поля $E=\widetilde{E} E_{0}=10 \mathrm{~V} / \mathrm{cm}, E_{0}=10^{4} \mathrm{~V} / \mathrm{cm}$. Таким образом, равномерное движение заряда (или полярона) в цепочке с параметрами ДНК возможно при напряженности электрического поля меньше значения $10 \mathrm{~V} / \mathrm{cm}$.

Для того чтобы быть уверенными, что мы наблюдаем равномерное движение полярона, а не участок его блоховских осцилляций, нужно провести вычисления продолжительностью хотя бы несколько блоховских периодов. Для моделирования равномерного движения полярона в ПБХ цепочке с параметрами, соответствующими параметрам цепочки ДНК, требуется брать очень длинные цепочки, и проводить очень длительные вычисления. Поэтому мы проводили моделирование движения полярона в цепочке при модельных параметрах, при которых требуется меньший объем вычислений. Мы провели вычисления для двух модельных значений параметра $\tau_{e}$ - отношение характерных времен эволюции волновой функции электрона и динамики возмущений в решетке. Были выбраны значения $\tau_{e}=4.5$ и 2.25 , для которых существенно меньше величины соответствующих блоховских периодов. Значения других параметров цепочки не изменялись.

Вычисления показали, что равномерное движения заряда по цепочке существует для небольших значений напряженности электрического поля. Причем заряд может двигаться равномерно на очень большие расстояния, на десятки тысяч сайтов. Для больших значений напряженности электрического поля равномерного движения заряда не наблюдается, заряд сразу теряет свою форму и, медленно продвигаясь в направлении поля, совершает блоховские осцилляции.

При переходе от режима равномерного движения заряда к колебательному режиму движения, при постепенном увеличении напряженности электрического поля, наблюдается характе́рный режим поведения заряда. В этих случаях для „промежуточных“ значений напряженности электрического поля после начала движения заряд (или полярон) движется по цепочке практически равномерно, почти сохраняя свою форму, затем полярон резко теряет свою форму и начинает совершать блоховские осцилляции. Период такого почти равномерного движения полярона до момента его перехода в колебательный режим движения заряда быстро возрастает c уменьшением напряженности электрического поля. В представленных примерах, продолжительность этого почти равномерного движения, наблюдалась в течение десятков блоховских периодов, и за это время заряд 
проходил тысячи сайтов цепочки. Очевидно, что для маленьких значений напряженности электрического поля, продолжительность такого „переходного“ равномерного движения заряда стремится к бесконечно большим значениям. Следовательно для небольших значений напряженности электрического поля это движение становится просто равномерным.

В рассмотренной нами модели ПБХ частоты блоховских осцилляций поляронов лежат в диапазоне $10-100 \mathrm{GHz}$, что представляет интерес для создания различных нанобиоэлектронных устройств. Так как характерная энергия рассмотренных нами „протяженных“ поляронов мала $(\sim 10-100 \mathrm{~K})$, то можно ожидать интересных особенностей в низкотемпературной области блоховских осцилляций, связанных с разрушением поляронных состояний.

Эти вопросы авторы планируют изучить в дальнейшем.

\section{Благодарности}

Работа выполнена с использованием вычислительных ресурсов Межведомственного суперкомпьютерного центра Российской академии наук (МСЦ РАН).

\section{Конфликт интересов}

Авторы заявляют, что у них нет конфликта интересов.

\section{Список литературы}

[1] Peyrard M., Cuesta-Lopez S., James G. // Nonlinearity. 2008. Vol. 21. P. 91-100. DOI: 10.1088/0951-7715/21/6/T02

[2] Zamora-Sillero E., Shapovalov A.V., Esteban F.J. // Phys. Rev. E. 2007. Vol. 76. P. 066603. DOI: 10.1103/PhysRevE.76.066603

[3] Starikov E.B. // Philosophical Magazine. 2005. Vol. 85. P. 3435-3462. DOI: 10.1080/14786430500157110

[4] Maniadis P., Kalosakas G., Rasmussen K.O., Bishop A.R. // Phys. Rev. E. 2005. Vol. 72. P. 021912.

DOI: 10.1103/PhysRevE.72.021912

[5] Komineas S., Kalosakas G., Bishop A.R. // Phys. Rev. E. 2002. Vol. 65. P. 061905. DOI: 10.1103/PhysRevE.65.061905

[6] Shigaev A.S., Ponomarev O.A., Lakhno V.D. // Chem. Phys. Lett. 2011. Vol. 513. P. 276-279.

DOI: $10.1016 /$ j.cplett.2011.07.080

[7] Berashevich J.A., Bookatz A.D., Chakraborty T. // J. Phys.: Condens. Matter. 2008. Vol 20. P. 035207. DOI:10.1088/0953-8984/20/03/035207

[8] Diaz E., Lima R.P.A., Dominguez-Adame F. // Phys. Rev. B. 2008. Vol. 78. P. 134303. DOI: 10.1103/PhysRevB.78.134303

[9] Modern Methods for Theoretical Physical Chemistry of Biopolymers. / Editors: Evgeni B. Starikov, James P. Lewis, Shigenori Tanaka. Elsevier. Amsterdam, 2006. 604 p.

[10] Long-Range Charge Transfer in DNA I. / Editors: Schuster, Gary B., Springer, 2004. 219 p. DOI: 10.1007/b84245

[11] Long-Range Charge Transfer in DNA II. / Editors: Schuster, Gary B., Springer, 2004. 245 p. DOI: 10.1007/b14032

[12] Fink H.W., Schönenberger C. // Nature. 1999. Vol. 398. P. 407.
[13] Баграев Н.Т., Чернев А.Л., Клячкин Л.Е., Маляренко А.М., Емельянов А.К., Дубина М.В. // ФТП. 2016. Т. 50. Вып. 9. C. $1230-1237$.

[14] Rakhmanova S.V., Conwell E.M. // J. Phys. Chem. B. 2001. Vol. 105. P. 2056. DOI: $10.1021 /$ jp0036285

[15] Lakhno V.D. // Int. Quantum. Chem. 2008. Vol. 108. P. 1970-1981. DOI: 10.1002/qua. 21717

[16] Nanobioelectronics - for Electronics, Biology and Medicine. / Eds. Offenhausser A., Rinaldi R. Springer, 2009. 338 p. DOI: $10.1007 / 978-0-387-09459-5$

[17] Eudres R.G., Cox D.L., Singh R.R.P. // Rev. Mod. Phys. 2004. Vol. 76. P. 195-214. DOI: 10.1103/RevModPhys.76.195

[18] Taniguchi M., Kawai T. // Physica E. 2006. Vol. 33. P. 1-12. DOI: 10.1016/j.physe.2006.01.005

[19] Porath D., Cuniberti G., Di Felice R. // Top. Curr. Chem. 2004. Vol. 237. P. 183-227. DOI: $10.1007 /$ b94477

[20] Lakhno V.D. // J. Biol. Phys. 2000. Vol. 26. P. 133. DOI: 10.1023/A:1005275211233

[21] Conwell E.M., Rakhmanova S.V. // Proc. Natl. Acad. Sci. 2000. Vol. 97. P. 4556. DOI: 10.1073/pnas.050074497

[22] Fialko N.S., Lakhno V.D. // Phys. Lett. A. 2000. Vol. 278. P. 108. DOI: $10.1016 / \mathrm{S} 0375-9601(00) 00755-6$

[23] Lakhno V.D., Korshunova A.N. // Math. Biol. Bioinf. 2010. Vol. 5. P. 1-29. DOI: $10.17537 / 2010.5 .1$

[24] Коршунова А.Н., Лахно В.Д. // ЖТФ. 2018. Т. 88. Вып. 9. C. 1312-1319. DOI: 10.21883/JTF.2018.09.46414.14-18 [Korshunova A.N., Lakhno V.D.] // Tech. Phys. 2018. Vol. 63. № 9. P. 1270-1276. DOI: $10.1134 / \mathrm{S} 1063784218090086$

[25] Holstein T. // Annals Phys. 1959. Vol. 8. P. 325-342. DOI: 10.1016/0003-4916(59)90002-8

[26] Holstein T. // Annals of Phys. 1959. Vol. 8. P. 343-389. DOI: 10.1016/0003-4916(59)90003-X

[27] Korshunova A.N., Lakhno V.D. // Physica E. 2014. Vol. 60. P. 206. DOI: 10.1016/j.physe.2014.02.025

[28] Lakhno V.D., Korshunova A.N. // Euro. Phys. J. B. 2007. Vol. 55. P. 85. DOI: 10.1140/epjb/e2007-00045-3

[29] Lakhno V.D., Korshunova A.N. // Eur. Phys. J. B. 2011. Vol. 79. P. 147. DOI: $10.1140 / \mathrm{epjb} / \mathrm{e} 2010-10565-2$

[30] Peyrard M., Bishop A.R. // Phys. Rev. Lett. 1989. Vol. 62. P. 2755-2758. DOI:10.1103/PhysRevLett.62.2755

[31] Dauxois T., Peyrard M., Bishop A.R. // Phys. Rev. E. 1993. Vol. 47. P. 684. DOI 10.1103/PhysRevE.47.684

[32] Peyrard M. // Europhys. Lett. 1998. Vol. 44. P. 271-277. DOI: $10.1209 / \mathrm{epl} / \mathrm{i} 1998-00469-9$

[33] Choi C.H., Kalosakas G., Rasmussen K.O., Hiromura M., Bishop A.R., Usheva A. // Nucleic Acids Res. 2004. Vol. 32. № 4. P. 1584-1590. DOI: 10.1093/nar/gkh335

[34] Кориунова А.Н., Лахно В.Д. // Математическая биология и биоинформатика. 2016. Т. 11. № 2. С. 141-158. DOI: $10.17537 / 2016.11 .141$

[35] Коршунова А.Н., Лахно В.Д. // Математическая биология и биоинформатика. 2017. Т. 12. № 1. С. 204-223. DOI: $10.17537 / 2017.12 .204$ 
[36] Коршунова А.Н., Лахно В.Д. // Математическая биология и биоинформатика. 2018. N. 13. № 2. C. 534-550. DOI: $10.17537 / 2018.13 .534$

[37] Лахно В.Д., Четвериков А.П. // Математическая биология и биоинформатика. 2014. Т. 9. № 1. С. 4-19. DOI: $10.17537 / 2014.9 .4$

[38] Chetverikov A.P., Ebeling W., Lakhno V.D., Shigaev A.S., Velarde M.G. // Eur. Phys. J. B. 2016. Vol. 89. P. 101. DOI 10.1140/epjb/e2016-60949-1 